\title{
Correlation between Sperm Motility and Morphology in the Success Rate of in Vitro Fertilization Procedure
}

\author{
Praditya Virza Ramadhan, ${ }^{1}$ Andri Rezano, ${ }^{2}$ Wiryawan Permadi ${ }^{3}$ \\ ${ }^{1}$ Faculty of Medicine Universitas Padjadjaran, ${ }^{2}$ Department of Anatomy and Cell Biology Faculty \\ of Medicine Universitas Padjadjaran, ${ }^{3}$ Department of Obstetric and Gynecology, Faculty of \\ Medicine, Universitas Padjadjaran/Dr. Hasan Sadikin General Hospital Bandung
}

\begin{abstract}
Background: In vitro fertilization (IVF) is one of the assisted reproductive technology that has been used in treating infertility. Unfortunately, not every IVF procedure achieves $100 \%$ success in fertilizing the oocyte. It has been studied that the percentage of normal motile sperm and percentage of normal sperm morphology play a key role in this process. This study was conducted to evaluate correlation between sperm motility and morphology in the success rate of IVF.

Methods: This study was an observational analytic involving medical records of infertile couples who were treated by IVF procedure in Dr.Hasan Sadikin General Hospital Bandung in 2009-2014. The variables that were used in this study were percentage of sperm motility, percentage of sperm morphology and fertilization rate.The collected data was analyzed using nonparametric Mann Whitney test and Spearman correlation test.

Result: This study showed that there was a significant difference between the fertilization rate group and sperm motility $(p=0,048)$ but there was no significant correlation between motility and the entire fertilization rate $(p=0,319)$. This study also indicated that there was no significant difference between fertilization rate group and sperm morphology $(p=0,232)$ and there was no significant correlation between sperm morphology and fertilization rate $(p=0,720)$.

Conclusions: Motility might has a role in determining the success rate of in vitro fertilization; meanwhile morphology does not have any role in determining the success rate of in vitro fertilization. [AMJ.2016;3(4):520-5]
\end{abstract}

Keywords: Fertilization rate, in vitro fertilization, sperm morphology, sperm motility

\section{Introduction}

Infertility is a condition affecting married couples who have failed to achieve conception after 12 months or more of having unprotected sex. ${ }^{1}$ Infertility occurs in $10-15 \%$ of couples. ${ }^{2}$ The first line therapy for infertility is by treating the cause of infertility. ${ }^{3}$ Assisted reproductive technologies (ART) is used if other medicines fail to treat the cause of infertility. ${ }^{3}$ In vitro fertilization (IVF) is one of the ART that is commonly used as it is safer than other assisted reproductive technologies. ${ }^{4}$ Fertilization in IVF is performed outside the female body and helps facilitating sperm to fertilize oocytes. Unfortunately, IVF is not always successful. ${ }^{5}$ A number of factors, from both males and females, influence the success of IVF.

One of the male factors that affects the success of IVF is the quality of sperm used. ${ }^{6,7}$ Sperm quality can be evaluated using semen analysis parameters. Parameters of semen analysis which are most frequently examined and are affiliated to fertility success are concentration, motility, and morphology. ${ }^{8}$ In vitro fertilization is considered to have a low success rate when sperm parameter results are below the World Health Organization (WHO) reference. ${ }^{9}$

However, it is still debatable whether sperm parameters are directly related to the success of in vitro fertilization. ${ }^{10}$ The main objective of this study was to assess correlation between the percentage of sperm morphology and

Correspondence: Praditya Virza Ramadhan, Faculty of Medicine, Universitas Padjadjaran, Jalan Raya BandungSumedang Km.21, Jatinangor, Sumedang, Indonesia, Phone: +6281224341516 Email: p.virza.r@gmail.com 
motility towards in vitro fertilization success.

\section{Methods}

This study used an analytic correlativecomparative method with a cross-sectional study design. The population of this study consisted of medical records of married couples who had used assisted reproductive technology in the form of IVF at Aster Fertility Clinic, Dr. Hasan Sadikin General Hospital Bandung, in the period of 2009-2014. Samples were taken from population using total sampling method. The sample was divided into two types. The first samples (N1) were taken to analyze correlation between sperm motility and fertilization success rate analysis and the other samples (N2) were taken to analyze correlation between sperm morphology and fertilization success rate analysis. The samples was also arbitrarily divided into 2 groups based on available data precentage of their fertilization success rate; high fertilization success rates $(\geq 50 \%)$ and low fertilization success rates $(<50 \%)$.

Medical records were compiled based on the inclusion and exclusion criteria. The inclusion criteria was age of female partner $<35$ years old, age of female partner $\geq 35$ years old with a Follicle Stimulating Hormone (FSH) rate $<10 \mathrm{mIU} / \mathrm{ml}$ on the $3^{\text {rd }}$ day of hormone therapy. The exclusion criteria was an FSH rate $\geq 10 \mathrm{mIU} / \mathrm{ml}$ on the third day of hormone therapy as this may cause a decrease in quality of the ovarian reserve, ${ }^{2}$ and incomplete data in medical records on the percentage of sperm motility, percentage of sperm morphology, number of oocytes collected and the number of successful fertilized oocytes.

Variables in this study were the percentages of sperm motility, sperm morphology, and fertilization success rate. The percentage of sperm motility was defined as the percentage of sperm that moved progressively against the total number of sperm. According to WHO, sperm motility below $40 \%$ was considered low. The percentage of sperm morphology was defined as the percentage of sperm with normal morphology as compared to the total number of sperm. According to WHO, sperm morphology below 4\% was considered low. Motility and morphology were assessed using computer aided-sperm analysis (CASA). The percentage of fertilization success was determined by comparing the total number of fertilized ovum, defined by formation of 2 pronuclei, with the total number of ovum collected. The percentage of successful fertilization was then categorized into low fertilization success $(<50 \%)$ and high fertilization success $(\geq 50 \%)$.

Data was presented in the form of averages $\pm \mathrm{SD}$ if the data was normally distributed; whereas, the data was presented in the form of medians and ranges between minimummaximum if data was not normally distributed. Nonparametric Mann Whitney Test was used to compare 2 groups of fertilization success

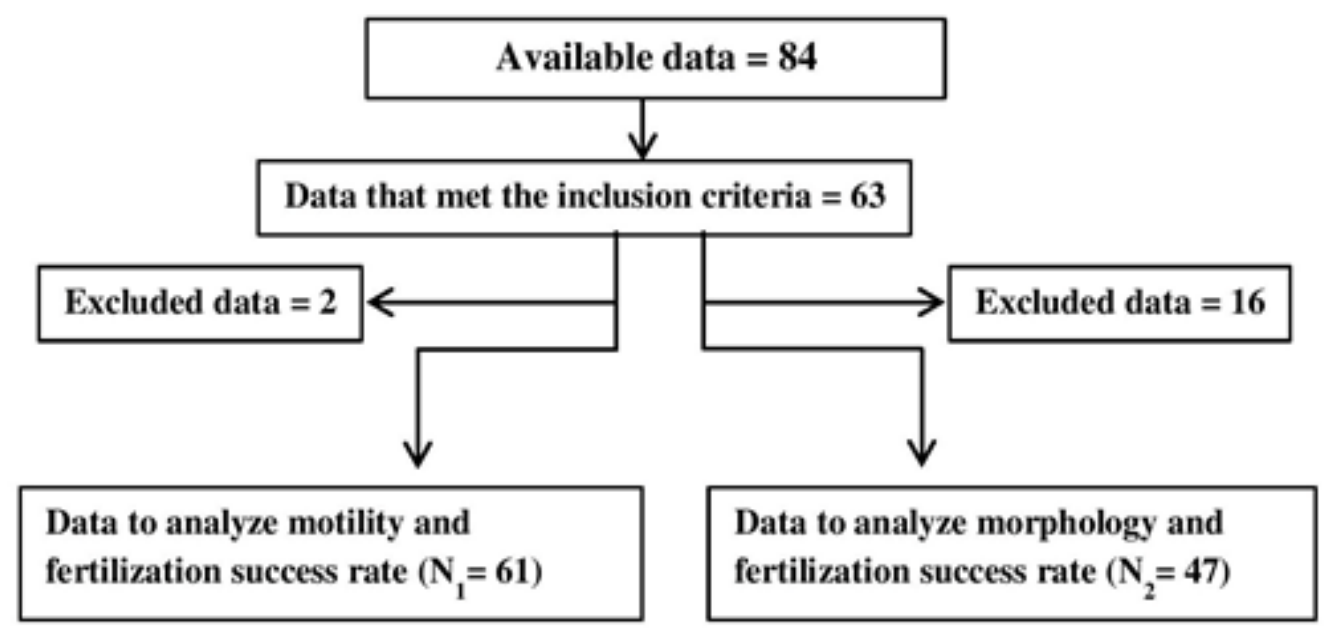

Figure 1 Sample Selection Process 
rates. Spearman Correlation Test was used to analyze the correlation between the percentage of sperm morphology and fertilization success rate and the same test was also used to analyze the correlation between the percentage of sperm motility and fertilization success rate.

This study had been approved by the Health Research Ethic Committee Dr. Hasan Sadikin General Hospital Bandung.

\section{Results}

Total of 84 medical records obtained, 63 medical records met the inclusion criteria. From 63 which met the inclusion criteria, there were 2 and 16 exluded data for $\mathrm{N} 1$ and N2 consecutively (Figure 1). Female partners who did not have data on FSH levels but were below the age of 35 years old were included in this study as female who were younger than 35 usually have FSH rate $<10 \mathrm{mIU} / \mathrm{ml}$ on the 3rd day of hormone therapy. ${ }^{11}$

In samples that were used to analyze correlation between motility and fertilization success rate, female factors were the most frequent cause of the infertility. Fallopian tube disorders were the most frequent infertilty disorder followed by endometriosis and asthenozoospermia.

In sampels that were used to analyze correlation between morphology and fertilization success rate, female factors were the most frequent cause of the infertility. Fallopian tube disorders were the most frequent infertilty disorder followed by asthenozoospermia and endometriosis.

Analysis showed that there was a significant difference $(p=0,048)$ between fertilization success rate group and the percentage of sperm motility, but eventhough the sperm motility was normal $(>40 \%)$ the fertilization success rate could still be low $(<50 \%)$ (Table 3). However, there was no significant difference $(p=0,232)$ between fertilization success rate group and the percentage of sperm morphology (Table 4).

Based on the correlation test, there was no significant correlation $(\mathrm{p}=0,319)$ between the percentage of sperm motility and the fertilization success. Based on the correlation test, there was no significant correlation $(p=0,720)$ between the percentage of sperm morphology and the fertilization success (Table 4).

Table 1 Sample Characteristics in Correlation between Motility and Fertilization Success Rate

\begin{tabular}{lc}
\hline \multicolumn{1}{c}{ Characteristics } & Total \\
\hline Female age & $35(25-44)^{*}$ \\
Male age & $37+-6^{* *}$ \\
Total sample & 61 \\
Factors affecting infertility & \\
$\quad$ Female & 39 \\
Male & 9 \\
Male and Female & 6 \\
Unexplained & 7 \\
Infertility disorders & \\
Adenomyosis & 3 \\
Endometriosis & 9 \\
Fallopian Tube disorders & 32 \\
Ovulation disorders & 3 \\
Asthenozoospermia & 9 \\
Teratoozoospermia & 6 \\
Unexplained & 7 \\
\hline
\end{tabular}

Note: *median and range (minimum-maximum)**average \pm standard deviation 
Table 2 Sample Characteristics in Correlation between Morphology and Fertilization Success Rate

\begin{tabular}{lc}
\multicolumn{1}{c}{ Characteristics } & Total \\
\hline Female age & $34 \pm 4^{*}$ \\
Male age & $37 \pm 6^{*}$ \\
Total sample & 47 \\
Factors causing infertility & 28 \\
$\quad$ Female & 9 \\
Male & 6 \\
Male and Female & 4 \\
Unexplained & \\
Infertility disorders & 3 \\
Adenomyosis & 7 \\
Endometriosis & 26 \\
Fallopian Tube disorders & 3 \\
Ovulation disorders & 8 \\
Asthenozoospermia & 6 \\
Teratoozoospermia & 4 \\
Unexplained &
\end{tabular}

Table 3 Comparison between Fertilization Success Rate Groups with Sperm Motility and Morphology Percentage

\begin{tabular}{|c|c|c|c|}
\hline \multirow{2}{*}{ Sperm Parameters } & \multicolumn{2}{|c|}{ Fertilization Success Rate } & \multirow{2}{*}{ p-value } \\
\hline & $<50 \%$ & $\geq 50 \%$ & \\
\hline Motility (\%) & $60(37-70)^{* * *}$ & $50(30-96)$ & $\mathrm{p}=0,048^{*}$ \\
\hline Morphology (\%) & $13(2-41)^{* * *}$ & $17(2-41)$ & $\mathrm{p}=0,232^{* *}$ \\
\hline
\end{tabular}

Note: *Mann-Whitney u-test, ${ }^{* *}$ Independent t-test, ${ }^{* * *}$ median and range (minimum-maximum)

\section{Discussion}

Theoretically, the difference between IVF and the natural fertilization process is the environment where fertilization takes place; however, the ability of sperm to penetrate the ovum is still required. ${ }^{6}$ The results from this study showed that there was a significant difference between the fertilization success rate group and the percentage of sperm motility (Table 3). This result was similar to a study by Langlois et al. ${ }^{8}$ Low in vitro fertilization success rates can be attributed to the percentage of sperm motility that is below the WHO reference $(<40 \%)^{4,8}$

Nonetheless, in this study, low fertilization success rates occurred in males with a sperm motility percentage above $40 \%$, and high

Table 4 Correlation between Sperm Motility and Morphology Percentage with Fertilization Success Rate

\begin{tabular}{lcc}
\hline Sperm Parameters & Fertilization Success Rate & Correlation Coefficient \\
\hline Motility (\%) & $\mathrm{p}=0,319^{*}$ & $-0,130$ \\
Morphology (\%) & $\mathrm{p}=0,720^{*}$ & 0,054 \\
\hline
\end{tabular}

Note: *Spearman correlation 
fertilization success rates can occur in males with a sperm motility percentage below $40 \%$ (Table 3). This showed that the number from a parameter analysis was not the only factor that affects fertilization success rates. Aside from a parameter analysis, a deeper evaluation on sperm function should be conducted. . $^{4,6,7}$ Other studies also suggested that the total number of motile sperm has more influence as compared to the percentage of motile sperm..$^{5,12}$ Nevertheless, there was no significant correlation between the percentage of sperm motility and fertilization success rate (Table 4) and this result was in contrast to the study by Langlois et al. ${ }^{8}$ This could be due to an uneven distribution of samples and an inadequate sample size in this study.

Based on the analysis results, there was a significant difference between the percentage of sperm morphology and fertilization success rates group (Table 3) and no significant correlation between the percentage of sperm morphology and fertilization success rate (Table 4). Low and high fertilization success rates occurred in males with a sperm morphology percentage of $41 \%$ (Table 3 ).

Low and high fertilization success rates can occur in males having a sperm morphology percentage below the WHO reference $(<4 \%)$. These results were contradict with studies by Langlois et al. ${ }^{8}$ and van der Westerlaken et al. ${ }^{13}$ which stated that the percentage of sperm morphology influences fertilization success rates in vitro because abnormalities in sperm morphology influences the ability of sperm to penetrate an oocyte.

On the other hand, studies by Lewis, ${ }^{10}$ Brita Söderlund and Lundin, ${ }^{14}$ and Ghirelli-Filho et al. ${ }^{15}$ concluded that sperm morphology did not influence fertilization success rates in IVF. The reason why the results were different could also because of the cause of the infertily in this study's sample (both female and male factors) and the other studies. This shows that sperm morphology is not an absolute factor in determining fertilization success rates.

In previous studies by Keegan et al. ${ }^{16}$ and van der Westerlaken et al. ${ }^{13}$ it was described that in vitro fertilization was still possible with a low percentage in sperm morphology as long as the percentage of sperm motility and the total motile sperm count was within the normal limits. ${ }^{13,16}$ Correlation between morphology and fertilization success rate is still debatable., .,10 $^{4}$

Technological advancement in this field of study, especially in the use of media and materials, has provided positive impact towards fertilization success rates in males with a low sperm morphology percentage. 16 The uncertainty of the influence of the percentage of sperm morphology can be attributed to the difficulty in having an objective evaluation in determining the total percentage of normal sperm morphology during semen analysis which may cause misinterpretation of results. ${ }^{14,15}$

The conclusion of this study is that the percentage of sperm motility influences the fertilization success rate. However, this study has not yet established relationship between the percentage of sperm motility and fertilization success rate whereas, the percentage of sperm morphology does not influence or has a significant relationship towards fertilization success rate.

This study had a number of limitations. The total number of samples collected did not meet the minimum sample size. Other functional sperm factors, which are rarely examined such as sperm DNA fragmentation, acrosin activity, and sperm chromatin conditions were not controlled and could influence fertilization success. Previous studies reported that these functional factors highly influence the success of sperms to fertilize ovum. It recommended to use a larger sample size and investigate other sperm factors in further study.

\section{References}

1. Zegers-Hochschild F, Adamson GD, de Mouzon J, Ishihara O, Mansour R, Nygren K, et al. International committee for monitoring assisted reproductive technology (ICMART) and the world health organization (WHO) revised glossary of art terminology, 2009. Fertil Steril. 2009;92(5):1520-4.

2. Fritz MA, Speroff L. Clinical gynecologic endocrinology and infertility. $8^{\text {th }}$ ed. Philadelphia: Wolters Kluwer Health/ Lippincott Williams \& Wilkins; 2011.

3. Nieschlag E, Behre HM, Nieschlag S. Andrology: male reproductive health and dysfunction. $3^{\text {rd }}$ ed. Berlin: Springer; 2010.

4. Chen X, Zhang W, Luo Y, Long X, Sun X. Predictive value of semen parameters in in vitro fertilisation pregnancy outcome. Andrologia. 2009;41(2):111-7.

5. Tournaye $\mathrm{H}$. Male factor infertility and art. Asian J Androl. 2012;14(1):103-8.

6. Aitken R. Sperm function tests and fertility. Int J Androl. 2006;29(1):69-75.

7. Simon L, Lewis SE. Sperm DNA damage or progressive motility: which one is the 
better predictor of fertilization in vitro?. Syst Biol Reprod Med. 2011;57(3):133-8.

8. Langlois MR, Oorlynck L, Vandekerckhove F, Criel A, Bernard D, Blaton V. Discrepancy between sperm acrosin activity and sperm morphology: Significance for fertilization in vitro. Clin Chim Acta. 2005;351(1):1219.

9. WangJ, Sauer MV. In vitro fertilization (IVF): a review of 3 decades of clinical innovation and technological advancement. Ther Clin Risk Manag. 2006;2(4):355-64.

10. Lewis SE. Is sperm evaluation useful in predicting human fertility? Reproduction. 2007;134(1):31-40.

11. Gardner DK, Weissman A, Howles CM, Shoham Z. Textbook of assisted reproductive techniques. $4^{\text {th }}$ ed. London: Informa Healthcare; 2012.

12. Wiser A, Ghetler Y, Gonen O, Piura E, Berkovits A, Itskovich A, et al. Reevaluation of post-wash sperm is a helpful tool in the decision to perform in vitro fertilisation or intracytoplasmic sperm injection. Andrologia. 2012;44(2):73-7.

13. van der Westerlaken $L$, Naaktgeboren $\mathrm{N}$, Verburg $\mathrm{H}$, Dieben S, Helmerhorst FM. Conventional in vitro fertilization versus intracytoplasmic sperm injection in patients with borderline semen: a randomized study using sibling oocytes. Fertil Steril. 2006;85(2):395-400.

14. Söderlund B, Lundin K. Choosing fertilization method by analyzing sperm morphology or by performing swim-up preparation. Acta Obstet Gynecol Scand. 2006;85(3):306-11.

15. Ghirelli-Filho M, Mizrahi FE, Pompeo ACL, Glina S. Influence of strict sperm morphology on the results of classic in vitro fertilization. Int Braz J Urol. 2012;38(4):519-28.

16. Keegan BR, Barton S, Sanchez X, Berkeley AS, Krey LC, Grifo J. Isolated teratozoospermia does not affect in vitro fertilization outcome and is not an indication for intracytoplasmic sperm injection. Fertil Steril. 2007;88(6):1583-8. 